\title{
COMPORTAMIENTO FRENTE A LA SEQUÍA DE PLANTAS REBROTADAS TRAS TALA EN BOSQUE MEDITERRÁNEO EL CASO DE Quercus ilex
}

Fleck, I. ${ }^{1.2}$, Aranda. $X^{1,3}$ y Peña-Rojas, K. ${ }^{1.4}$

\section{RESUMEN}

Se estudió la conducta ecofisiológica en respuesta a la sequia de plantas de encina (Quercus ilex L.) intactas (controles) y plantas rebrotadas tras tala de la misma especie. El experimento se realizó a lo largo de un año en la Sierra de Collserola, Barcelona, España utilizando diversas parcelas de bosque con diferente disponibilidad de agua en el suelo. Se midieron las relaciones hidricas, las tasas de intercambio gaseoso foliar, fluorescencia de las clorofilas, composición química y caracteristicas estructurales en los dos tipos de plantas.

Cuando la disponibilidad hidrica del suelo fue elevada, la fotosintesis fue similar en rebrotes y controles, a pesar de la mayor hidratación de las hojas y el mayor contenido hidrico relativo al mediodia en las plantas rebrotadas. Bajo sequia moderada, la menor demanda hídrica de los rebrotes, debida a su menor área foliar total, se reflejó en un contenido hidrico relativo constante y mayores conductancias estomáticas y fotosintesis. El cierre estomático fue el limitante de la fotosintesis en controles. Bajo sequia severa, se registró una limitación no estomática de la fotosintesis, menos pronunciada en rebrotes, que se reflejaba en los parámetros de fluorescencia de las clorofilas: ambos grupos presentaban menores valores de rendimiento cuántico del fotosistema II y mayor disipación térmica de la energia absorbida comparados con una disponibilidad hídrica elevada, especialmente en el caso de los controles. En esta etapa, se observó un cambio en estructura y composición quimica foliar de la vegetación rebrotada en respuesta a la sequia severa. En estas plantas, se observaron cambios en las respectivas contribuciones de la densidad y grosor de la hoja en el peso especifico foliar. Una menor densidad en las hojas, relacionada con menores contenidos de hemicelulosa, celulosa y lignina, conllevó a una mayor conductancia para el $\mathrm{CO}_{2}$ en el mesófilo y como resultado una mayor capacidad fotosintética favoreciendo el crecimiento en las plantas rebrotadas respecto a las intactas.

Palabras clave: Bosque Mediterráneo, Quercus ilex, Rebrote, Sequia.

1-Departamento de Biologia Vegetal, Universidad de Barcelona, España 


\section{SUMMARY}

The ecophysiological behaviour in response to drought of undisturbed holm-oak (Quercus ilex L., controls) and resprouts after clear-cut of the same species is studied. Experiments were conducted throughout one year in Serra de Collserola, Barcelona, España at a number of forest sites differing in soil water availability. Water status, leaf gas-exchange rates, chlorophyll fluorescence, chemical composition and structural attributes on both plant types were measured.

When soil water availability was high, photosynthesis was similar in resprouts and controls, despite higher midday leaf hydration and relative water content in resprouts. In moderate drought, the lower water demand of resprouts, resulting from their lower total leaf area, accounts for the constant relative water content and high stomatal conductance and photosynthesis. Stomatal closure was found to limit photosynthesis in controls, and in severe drought, nonstomatal limitation of photosynthesis was also greater than in resprouts. This limitation was reflected in chlorophyll fluorescence parameters: both groups presented lower values of photosystem II quantum yield and higher energy dissipation as heat, especially in controls. At this stage, the foliar structure and chemical composition of the resprouting vegetation had changed in response to severe drought. In these plants, changes in the respective contributions of leaf density and thickness to the leaf mass per area ratio were observed. A lower leaf density, related to lower hemicellulose, cellulose and lignin content, accounted for the higher mesophyll conductance to $\mathrm{CO}_{2}$, which resulted in greater photosynthetic capacity and growth in resprouts than in controls.

Key words: Mediterranean Forest, Quercus ilex, Coppice, Drougth. 


\section{INTRODUCCIÓN}

Las condiciones de incremento de temperatura asociadas al cambio climático (Houghton et al., 2001) aumentarán el riesgo de sequías siendo probablemente el área mediterránea las zona con mayor impacto (Chaves et al., 2003). Sus efectos serán patentes en la fisiología de las especies vegetales. Asimismo el aumento de la frecuencia e intensidad de los incendios forestales (Mouillot et al., 2002) podría reducir la capacidad de rebrote y recuperación de las especies (Kruger y Reich, 1997).

Una de las especies más comunes en la cuenca mediterránea es la encina (Quercus ilex L.), poseedora de raices profundas y una gran capacidad de rebrote a partir de los órganos subterráneos tras sufrir una perturbación (incendio forestal, tala, herbivoria). Los rebrotes originados tras incendio o tala poseen caracteristicas comunes, mostrando durante los primeros años unas tasas fotosintéticas y de crecimiento muy superiores a las de individuos que no sufrieron perturbación (Fleck et al., 1996, Fleck et al., 1998). Este hecho es debido a una serie de factores, entre ellos la utilización de reservas subterráneas y la mayor disponibilidad de agua y/o nutrientes (Saruwatari y Davis, 1989) debido a la alteración de la relación vàstago/raiz (disminución de la parte aérea asociada a una gran masa radicular).

Bajo condiciones de disponibilidad hídrica limitada, el cierre estomático reduce la disponibilidad de $\mathrm{CO}_{2}$ en el mesófilo del cloroplasto que conlleva a una disminución de la fotosintesis especialmente al mediodia (Tenhunen et al., 1987). Bajo estrés hidrico moderado, el cierre estomático se considera el principal factor limitante e la fotosintesis mientras que al incrementarse la sequia aparecen limitantes no estomáticos (Flexas et al., 2002; Lawlor y Cornic, 2002; Peña-Rojas et al., 2004). La conductancia al $\mathrm{CO}_{2}$ dentro de la hoja, denominada conductancia del mesófilo $\left(\mathrm{g}_{\text {mes }}\right.$ ) se considera particularmente importante (Lloyd et al., 1992; Loreto et al., 1994; Epron et al., 1995; Kogami et al., 2001, Niinemets et al., 2005).

La disponibilidad hídrica juega también un papel modificando la estructura foliar (Niinemets, 2001), que a su vez afecta al intercambio gaseoso. La densidad foliar afecta la difusión de $\mathrm{CO}_{2}$ a los sitios de carboxilación que puede ser particularmente significante en esclerófilos (Loreto et al., 1994; Terashima et al., 2001).

\section{OBJETIVOS}

A fin de predecir los efectos de cambios ambientales sobre el proceso fotosintético, la productividad y la capacidad de supervivencia de los encinares, se debe conocer el funcionamiento de dicha especie en situaciones de baja disponibilidad hídrica. Por ello, los objetivos del presente trabajo fueron analizar durante la sequia los cambios funcionales y estructurales que tienen lugar en hojas de encina de individuos no perturbados (controles) y en rebrotes originados después de tala. En particular se estudió la contribución de la conductancia del mesófilo al $\mathrm{CO}_{2}\left(\mathrm{~g}_{\text {mes }}\right)$ limitando la fotosintesis. El estudio se realizó desde invierno (sequia moderada) hasta verano (sequia severa). Asimismo se compararon rebrotes con individuos controles crecidos en la misma parcela o bien en una zona no perturbada. La transpiración del dosel y por tanto la disponibilidad hidrica diferirá entre ambas áreas. 


\section{MATERIAL Y MÉTODO}

\section{Localización del Estudio}

El estudio se realizó a lo largo de un año en Can Coll, Sierra de Collserola, Barcelona, España $\left(41^{\circ} 28^{\prime} 28^{\prime \prime} \mathrm{N}, 2^{\circ} 7^{\prime} 32^{\prime \prime} \mathrm{E}\right)$. El bosque, de 35 años de edad está dominado por Quercus ilex y Pinus halepensis.

\section{Caracteristicas Climáticas}

El clima es Mediterráneo con inviernos fríos, primavera y otoño son lluviosos y los veranos secos y calurosos. Las características climáticas durante el estudio se muestran en el Cuadro $\mathrm{N}^{\circ} 1$.

\section{Cuadro $\mathrm{N}^{\circ} 1$}

\section{DATOS CLIMATOLÓGICOS DE LA ESTACIÓN METEOROLÓGICA MÁS PRÓXIMA (2 km) CORRESPONDIENTES A LOS TREINTA DIAS ANTERIORES A LA TOMA DE DATOS}

\begin{tabular}{|l|c|c|c|}
\hline \multirow{2}{*}{ Parámetro } & Invierno & Primavera & Verano \\
\cline { 2 - 4 } & $\mathbf{1 5 / 0 2 - 1 5 / 0 3}$ & $\mathbf{1 5 / 0 5 - 1 5 / 0 6}$ & $\mathbf{1 5 / 0 7 - 1 5 / 0 8}$ \\
\hline Temperatura media $\left({ }^{\circ} \mathrm{C}\right)$ & 11,6 & 20,0 & 24,0 \\
\hline Temperatura máxima media $\left({ }^{\circ} \mathrm{C}\right)$ & 17,9 & 26,8 & 31,8 \\
\hline Temperatura minima media $\left({ }^{\circ} \mathrm{C}\right)$ & 8,0 & 15,7 & 19,0 \\
\hline Precipitación acumulada $(\mathrm{mm})$ & 20,8 & 39,1 & 5,8 \\
\hline $\begin{array}{l}\text { Velocidad media del viento }(\mathrm{m} / \mathbf{S}) \text { y } \\
\text { dirección }\end{array}$ & $5,1(\mathrm{SW})$ & $4,2(\mathrm{SW})$ & $4,9(\mathrm{SW})$ \\
\hline Presión atmosférica $(\mathrm{HPa})$ & 1028 & 1020 & 1019 \\
\hline Déficit de presión de vapor $(\mathrm{KPa})$ & 1,04 & 1,14 & 2,05 \\
\hline
\end{tabular}

\section{Diseño}

Se seleccionó una parcela $(400 \times 280 \mathrm{~m})$ a $140 \mathrm{msnm}$ y orientada $\mathrm{N}-\mathrm{NE}$, que se mantiene habitualmente con una baja densidad de plantas. En ella se eligieron al azar 10 plantas que fueron taladas en invierno a $15 \mathrm{~cm}$ del nivel del suelo (Rebrotes, R), y 5 plantas más, no taladas, se utilizaron como controles de dicha parcela (Controles, C). En el bosque circundante, se eligieron al azar 4 individuos de $Q$. ilex próximos a la parcela, que fueron designados como CF (Control Forest, controles bosque). Las caracteristicas morfológicas de los árboles antes de talarlos fueron: $5,9 \pm 0.3 \mathrm{~cm}$ diámetro a la altura del pecho (DBH), $4,7 \pm 0.2$ $\mathrm{m}$ altura media, $1,4 \pm 0.2 \mathrm{Kg}$ biomasa foliar media.

\section{Variables Analizadas}

- Intercambio Gaseoso y Fluorescencia de las Clorofilas

Se utilizó el sistema de intercambio gaseoso portátil LI-6200 (Li-Cor Inc., Lincoln, 
NE, USA) para medidas puntuales al mediodia. En cada estación se realizaron 10 curvas de respuesta de la asimilación de $\mathrm{CO}_{2}$ respecto de concentración intercelular de $\mathrm{CO}_{2}\left(\mathrm{~A} / \mathrm{C}_{1}\right.$ ) por tratamiento mediante el sistema de intercambio da gases LI-6400 (Li-COR, Lincoln, Nebraska, USA). Las condiciones en la cubeta foliar variaron según la estación.

La densidad de flujo fotónico fotosintético se estableció como $600 \mu \mathrm{mol} \cdot \mathrm{m}^{2} \cdot \mathrm{s}^{-1}$, que es saturante en estas condiciones (Peña-Rojas et al., 2004) y la concentración ambiental de $\mathrm{CO}_{2}$ estuvo comprendida entre 50 y $800 \mu \mathrm{mol} \cdot \mathrm{mol}^{-1} \mathrm{El}$ análisis de las curvas permitió la determinación de $A_{\max }$, fotosíntesis neta a $C_{\text {, y }}$ PPFD saturante; $V_{c, \text { max }}$, velocidad máxima de carboxilación de la Rubisco; $J_{\max }$, transporte máximo de electrones que contribuye a la regeneración de la ribulosa bifosfato (RuBP), $I_{s}$, limitación estomática de la fotosintesis $\left(I_{s}(\%)=100 \times\left(1-\left(A_{\mathrm{s}} \mathrm{A}_{\mathrm{sal}}\right)\right.\right.$ ): $A_{\text {sat }}$, fotosintesis neta bajo luz saturante y $C_{i}=350 \mu \mathrm{mol} \cdot \mathrm{mol}-1$ ) (Farquhar y Sharkey, 1982; McMurtrie y Wang, 1993).

Los componentes de la fluorescencia de las clorofilas se cuantificaron mediante un fluorimetro modulado (Mini-PAM Photosynthesis Yield Analyzer, Walz, Effeltrich, Alemania) en las mismas hojas utilizadas en las mediciones de intercambio gaseoso tal como se describe en Fleck et al. (1998). Los parámetros $F_{m}, F_{m}^{\prime}, F_{0}$ y $F_{v}$ se determinaron según Kooten y Snel (1990). La eficiencia del PSII $\left(\Phi_{P S I I}\right)$ y el rendimiento cuántico máximo al mediodia $\left(F / F_{m}\right)$ se determinaron según Genty et al. (1989). Los parámetros $F^{\prime}$, $F^{\prime}$, y quenching fotoquimico $\left(q_{p}\right.$. relacionado con el número de centros abiertos) se estimaron según Oxborough y Baker (1997). El quenching no fotoquimico (NPQ) se calculó como $\left(\left(\mathrm{F}_{m} / \mathrm{F}_{m}^{\prime}\right)-1\right)$. La adaptación en oscuridad fue de minimo 20 minutos.

La conductancia del mesófilo $\left(\mathrm{g}_{\mathrm{mes}}\right)$ se estimó a partir de las medidas combinadas de intercambio de gases (LiCor 6400) y de fluorescencia de las clorofilas según Epron et al. (1995) y Flexas et al. (2002).

\section{- Contenido Hídrico Relativo y Parámetros Anatómicos}

El contenido hidrico relativo (RWC) se determinó calculando: $R W C=[(f . w t-d . w t) /(f s$. wt-d.wt) 100], f.wt peso fresco; fs.wt peso saturado (tras rehidratar las muestras durante $24 \mathrm{~h}$ en oscuridad) y d.wt peso seco (tras secar las muestras en estufa a $65^{\circ} \mathrm{C}$ hasta peso constante). Se determinaron en invierno y verano en las mismas plantas utilizadas para mediciones de intercambio de gases: el peso especifico foliar, LMA = (d.wt/LA), y sus componentes (f.wt/ LA) y [(d.wt/f.wt)-100], como indicadores del grosor foliar (T) y de la densidad foliar (D), respectivamente (Dijkstra, 1989).

\section{-Composición Química Foliar}

Se molieron muestras de hojas en un Molino Cyclotec 1093 Sample Mill (Tecator, Höganäs, Suecia). Posteriormente se escanearon en un espectrofotómetro de reflectancia en el infrarojo cercano (NIR Systems 6500. Foss NIR Systems, Inc, Silver Spring, MD). Se utilizó una base de datos espectral a partir de 25 especies leñosas mediterráneas tal como se describe en Peña-Rojas et al. (2005) 


\section{RESULTADOS Y DISCUSIÓN}

Durante el estudio se observaron diferencias funcionales y estructurales entre rebrotes y plantas control, especialmente en el periodo de sequia. Los valores de los parámetros hídricos determinados reflejaron las diferencias en disponibilidad hídrica de los distintos tipos de plantas (tratamientos) (Figura $\mathrm{N}^{\circ}$ 1).

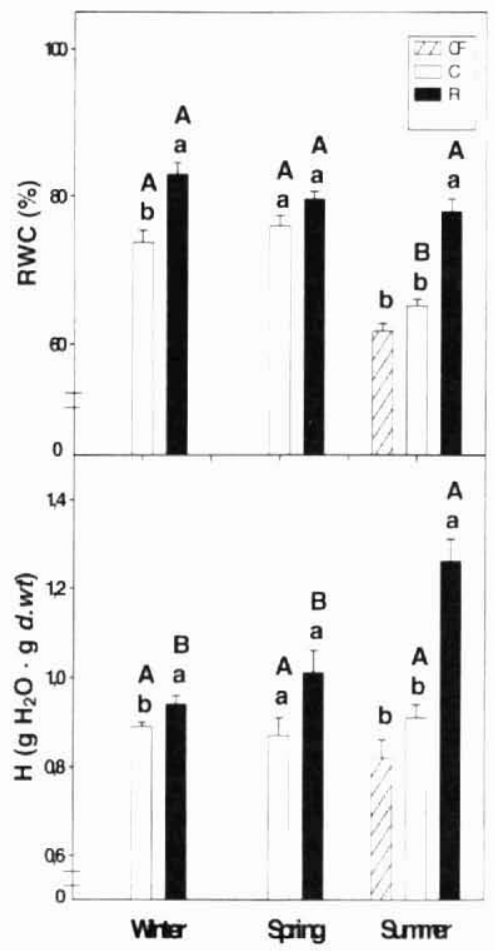

Cada valor representa la media \pm SE de 30 medidas.

Las diferencias significativas ( $\mathrm{p} \leq 0.05)$ se indican mediante letras distintas (tratamiento $(a, b)$, estación $(A, B)$ ).

Figura $\mathbf{N}^{\circ} 1$

CONTENIDO HIDRICO RELATIVO (RWC) E HIDRATACIÓN (H) AL MEDIODIA (CF CONTROL BOSQUE; C CONTROL Y R, REBROTES)

Al mediodia, los rebrotes mostraron mayor contenido hidrico (RWC) e hidratación (H) que los controles en invierno y verano, hecho relacionado con su menor relación parte aéreal parte subterránea (Peña-Rojas et al., 2004, Fleck et al., 1998). A pesar de que el aumento en temperatura foliar y DPV en verano favorecieron una elevada transpiración en rebrotes (Figura $N^{\circ} 2$ ), el RWC se mantuvo a lo largo del año y fue superior al de los controles. Las diferencias entre $\mathrm{R}$ y $\mathrm{C}$ fueron iguales a las diferencias entre R y CF y en consecuencia no son debidas a la zona en que crecieron (talada o intacta) y sus presumibles diferencias en contenido de agua en el suelo, sino a las caracteristicas de los rebrotes. 


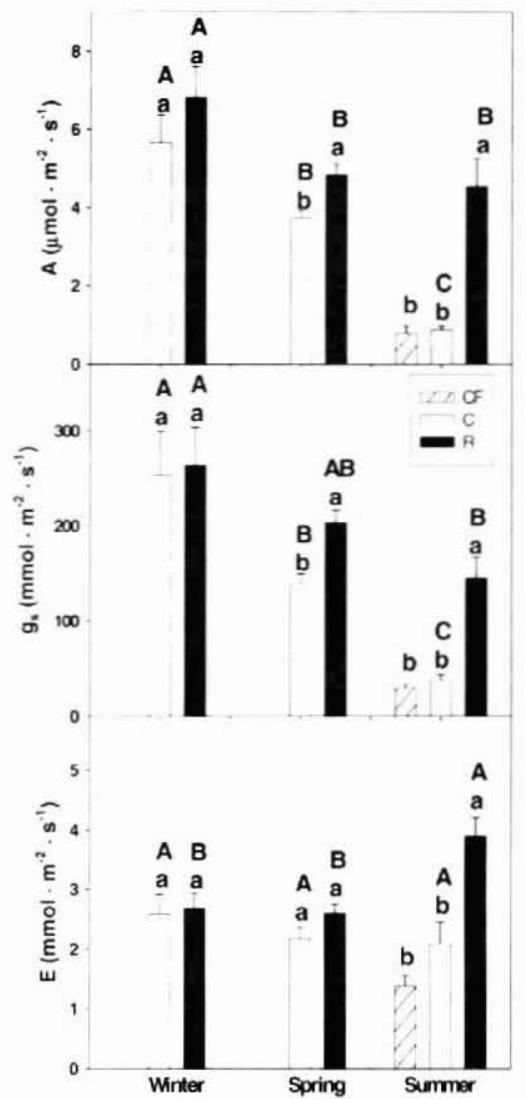

Cada valor representa la media \pm SE de 8 medidas.

Las diferencias significativas $(p \leq 0.05)$ se indican mediante letras distintas (tratamiento (a, b, c), estación (A, B)).

Figura $\mathrm{N}^{\circ} 2$

\section{VALORES AL MEDIODIA DE ASIMILACIÓN NETA DE $\mathrm{CO}_{2}$ (A), CONDUCTANCIA ESTOMÁTICA $\left(G_{\mathrm{S}}\right.$ ) Y TRANSPIRACIÓN (E), (CF CONTROL BOSQUE; C CONTROL Y R, REBROTES).}

Cuando la disponibilidad hidrica fue mayor (invierno), la fotosintesis fue semejante en los diferentes tipos de material, sin observarse ventaja de los rebrotes a pesar de mostrar mayor RWC e hidratación (Figura $\mathrm{N}^{\circ} 2$ ). Bajo un estrés hídrico moderado (primavera), la menor demanda hidrica de los rebrotes debida a su menor área foliar total implicó una mayor conductancia estomática que en los controles. El cierre estomático redujo la disponibilidad de $\mathrm{CO}_{2}$ conllevando una disminución de la fotosintesis. El estrés estival tuvo un efecto marcado reduciendo la fotosintesis un $50 \%$ en los controles y un $20 \%$ en los rebrotes. En resumen, controles y rebrotes evitaron la desecación mediante el cierre estomático, con lo que se produjo una limitación de la asimilación de $\mathrm{CO}_{2}$ pero se mantuvieron unos valores operativos de RWC $\mathrm{yH}$. 
En determinadas circunstancias, la absorción de luz puede exceder a la necesaria para la fotosintesis llevando a la fotoinhibición del aparato fotosintético en dicha especie (Méthy et al. 1996). El efecto de la sequia quedó reflejado en los parámetros de fluorescencia (Figura $N^{\circ} 3$ ). El $\Phi$ PSII, $F / F_{r i t}$ y el $q_{p}$ fueron menores en el verano. La disipación de la energia en forma de calor mediante el ciclo de las xantofilas, indicado por el parámetro NPQ fue mayor que en invierno en todos los tratamientos indicando una mayor saturación del aparato fotosintético. (DemmigAdams y Adams 1996). Asimismo, el NPQ en verano fue menor en rebrotes que en controles debido a su mayor sumidero fotosintético de electrones (mayor fotosintesis). Los valores de $\Phi_{\text {PSII, }}$ NPQ y $q_{p}$ en CF son indicativos de un estado del aparato fotoquimico más relajado en el ambiente sombreado. Los resultados de intercambio de gases y de fluorescencia de las clorofilas indicaron la existencia de limitantes no estomáticos de A durante la sequia severa, menos marcada en rebrotes, corroborados por los cambios observados en los parámetros derivados de las curvas $\mathrm{A} / \mathrm{C}_{i}\left(\right.$ Cuadro $\left.\mathrm{N}^{\circ} 2\right)$.

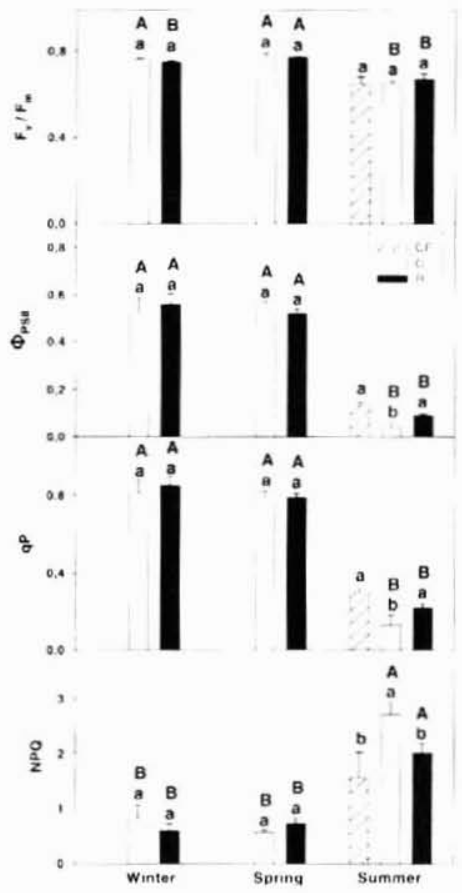

Cada vaior representa la media \pm SE de 8 medidas.

Las diferencias significativas ( $p \leq 0.05$ ) se indican mediante letras distintas (tratamiento ( $a, b, c)$. estación (A, B)).

Figura $\mathrm{N}^{\circ} 3$

VALORES AL MEDIODIA DE RENDIMIENTO CUÁNTICO MÁXIMO DEL PSII $\left(\mathrm{F}_{\checkmark} / \mathrm{F}_{\mathrm{N}}\right)$, EFICIENCIA DEL PSII (ФPSII), QUENCHING FOTOQUIMICO $\left(Q_{p}\right)$ Y QUENCHING NO FOTOQUIMICO (NPQ);

(CF CONTROL BOSQUE; C CONTROL Y R, REBROTES). 


\section{Cuadro $\mathrm{N}^{\circ} 2$}

ASIMILACIÓN NETA DE CO ${ }_{2}$ A C Y Y LUZ SATURANTE ( $A_{\text {Max }}$ ), VELOCIDAD MÁXIMA DE CARBOXILACIÓN DE LA RUBISCO $\left(V_{\text {C,MAX }}\right)$, TASA MAXIMA DE TRANSPORTE DE ELECTRONES QUE CONTRIBUYE A LAREGENERACIÓN DE LA RUBP $\left(J_{\text {MAX }}\right) Y$ LIMITACIÓN ESTOMÁTICA $\left(L_{s}\right)$ A PARTIR DE LAS CURVAS A/C, PARA LOS DISTINTOS TRATAMIENTOS (C, CONTROL; R, REBROTES) Y ESTACIONES

\begin{tabular}{|c|c|c|c|}
\hline & & 1 & \\
\hline & & C & $\mathbf{R}$ \\
\hline \multirow{2}{*}{$A_{\max }\left(\mu \mathrm{mol} \cdot \mathrm{m}^{2} \cdot \mathrm{s}^{-1}\right)$} & Invierno & $6.6 \pm 0.5^{a 8}$ & $7.2 \pm 0.3^{* 6}$ \\
\hline & Verano & $1.1 \pm 0.2^{\text {an }}$ & $3.4 \pm 0.5^{\text {ba }}$ \\
\hline \multirow{2}{*}{$V_{\text {cmax }}\left(\mu \mathrm{mol} \cdot \mathrm{m}^{2} \cdot \mathrm{s}^{-1}\right)$} & Invierno & $30.7 \pm 2.8^{\circ e}$ & $31.8 \pm 1.6^{n}$ \\
\hline & Verano & $13.5 \pm 4.0^{\mathrm{x}}$ & $29.9 \pm 2.3^{\text {ta }}$ \\
\hline \multirow{2}{*}{$\mathrm{J}_{\max }\left(\mu \mathrm{mol} \cdot \mathrm{m}^{-2} \cdot \mathrm{s}^{-1}\right)$} & Invierno & $63.4 \pm 4.9^{a t}$ & $66.8 \pm 2.8^{\mathrm{at}}$ \\
\hline & Verano & $17.1 \pm 4.1^{\mathrm{ma}}$ & $34.9 \pm 4.3^{\text {tha }}$ \\
\hline \multirow{2}{*}{$I_{s}(\%)$} & Invierno & $26.2 \pm 1.5^{\mathrm{m}}$ & $21.9 \pm 1.1^{\text {nh }}$ \\
\hline & Verano & $51.6 \pm 3.9^{\circ 8}$ & $37.5 \pm 3.0^{* 8}$ \\
\hline
\end{tabular}

Cada valor representa la media \pm SE de 10 réplicas. Las diferencias significativas $(p \leq 0.05)$ se indican mediante letras distintas (tratamiento $(a, b, c)$, estacion (A, B)).

Con la progresión de sequia de invierno a verano, se observaron cambios morfológicos: bajo disponibilidad hidrica baja las hojas tendieron a ser menores (LA), caracteristica frecuente en especies esclerófilas perennifolias (Gratani y Bombelli, 1999). La mejora hidrica en rebrotes se evidenció en hojas mayores. CF también mostró áreas mayores atribuibles a su ambiente menos soleado. Con mayor sequia se observaron LMA mayores: Ios $\mathrm{R}$ mostraron valores menores en verano. Mayores LMA en controles estuvieron asociados con tasas de $A$ menores de acuerdo con Gulias et al. (2003). 


\section{Cuadro $\mathrm{N}^{\circ} 3$}

COMPOSICIÓN QUIMICA FOLIAR. CF CONTROL BOSQUE, C CONTROL, R REBROTES, TNC CARBOHIDRATOS TOTALES NO ESTRUCTURALES

\begin{tabular}{|c|c|c|c|c|}
\hline & \multirow{2}{*}{$\mathrm{g} \cdot \mathrm{m}^{-2}$} & \multicolumn{3}{|c|}{ Tratamiento } \\
\hline & & CF & C & $\mathbf{R}$ \\
\hline E- & Invierno & - & $2.57 \pm 0.26^{a A}$ & $2.36 \pm 0.12^{\mathrm{aA}}$ \\
\hline Nitrógeno & Primavera & - & $2.20 \pm 0.07^{\mathrm{aA}}$ & $2.01 \pm 0.06^{\mathrm{aB}}$ \\
\hline 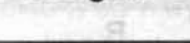 & Verano & $2.22 \pm 0.14^{a}$ & $2.05 \pm 0.10^{\mathrm{aA}}$ & $1.99 \pm 0.05^{\mathrm{aB}}$ \\
\hline \multirow{3}{*}{ Almidón } & Invierno & $\ldots$ & $5.03 \pm 1.93^{\mathrm{aA}}$ & $8.02 \pm 1.44^{\mathrm{aA}}$ \\
\hline & Primavera & $\ldots$ & $9.39 \pm 0.80^{\mathrm{aB}}$ & $9.28 \pm 0.51^{\mathrm{aA}}$ \\
\hline & Verano & $11.88 \pm 0.04^{\mathrm{a}}$ & $11.05 \pm 0.31^{\mathrm{aB}}$ & $10.57 \pm 0.33^{\mathrm{aA}}$ \\
\hline \multirow{3}{*}{$\begin{array}{l}\text { Azúcares } \\
\text { solubles }\end{array}$} & Invierno & -- & $11.45 \pm 2.61^{\mathrm{aA}}$ & $10.16 \pm 0.85^{\mathrm{aA}}$ \\
\hline & Primavera & -- & $11.40 \pm 0.91^{\mathrm{aA}}$ & $9.08 \pm 0.61^{\mathrm{bA}}$ \\
\hline & Verano & $11.66 \pm 0.63^{a}$ & $12.15 \pm 0.42^{\mathrm{aA}}$ & $9.51 \pm 0.28^{\mathrm{bA}}$ \\
\hline \multirow{3}{*}{ Lipidos } & Invierno & -- & $19.95 \pm 4.06^{\mathrm{aA}}$ & $17.14 \pm 1.82^{\mathrm{aA}}$ \\
\hline & Primavera & - & $22.30 \pm 1.07^{\mathrm{aA}}$ & $16.13 \pm 1.00^{\mathrm{bA}}$ \\
\hline & Verano & $24.33 \pm 1.25^{a}$ & $23.61 \pm 0.92^{\mathrm{aA}}$ & $18.68 \pm 0.57^{\mathrm{DA}}$ \\
\hline \multirow{3}{*}{ TNC } & Invierno & - & $16.48 \pm 4.54^{\mathrm{aA}}$ & $18.18 \pm 2.26^{\mathrm{aA}}$ \\
\hline & Primavera & - & $20.79 \pm 1.40^{\mathrm{aA}}$ & $18.36 \pm 0.84^{a A}$ \\
\hline & Verano & $23.54 \pm 0.59^{a}$ & $23.20 \pm 0.66^{\mathrm{aA}}$ & $20.09 \pm 0.47^{t . A}$ \\
\hline \multirow{3}{*}{ Hemicelulosa } & Invierno & $\ldots$ & $25.56 \pm 2.68^{\mathrm{aA}}$ & $23.85 \pm 1.01^{2 \mathrm{~A}}$ \\
\hline & Primavera & $\ldots$ & $26.01 \pm 0.85^{\text {aA }}$ & $22.45 \pm 0.68^{\mathrm{DA}}$ \\
\hline & Verano & $27.89 \pm 0.87^{a}$ & $26.96 \pm 0.71^{\mathrm{aA}}$ & $25.15 \pm 0.75^{a A}$ \\
\hline \multirow{3}{*}{ Celulosa } & Invierno & -- & $34.91 \pm 1.88^{\mathrm{aA}}$ & $30.01 \pm 1.76^{\mathrm{aA}}$ \\
\hline & Primavera & --. & $33.25 \pm 0.77^{\mathrm{aA}}$ & $30.02 \pm 0.96^{\mathrm{DA}}$ \\
\hline & Verano & $39.10 \pm 0.08^{a}$ & $33.96 \pm 0.56^{\mathrm{DA}}$ & $31.78 \pm 1.01^{\mathrm{bA}}$ \\
\hline \multirow{3}{*}{ Lignina } & Invierno & -- & $31.72 \pm 0.26^{\mathrm{aA}}$ & $24.11 \pm 1.85^{\mathrm{bA}}$ \\
\hline & Primavera & - & $26.66 \pm 0.67^{a \mathrm{~B}}$ & $22.76 \pm 0.77^{\mathrm{bA}}$ \\
\hline & Verano & $28.41 \pm 1.96^{a}$ & $24.81 \pm 0.99^{\mathrm{abB}}$ & $22.05 \pm 1.01^{\mathrm{bA}}$ \\
\hline
\end{tabular}

Los valores corresponden a la media \pm SE de 10 réplicas. Las diferencias significativas

$(p \leq 0.05)$ se indican mediante letras distintas (tratamiento a, b, c, estación A, B)

También se observó un contenido similar de $\mathrm{N}$ (Cuadro $\mathrm{N}^{\circ} 3$ ) en controles y rebrotes a pesar de las menores tasas fotosintéticas en C. Esta observación confirma que existe una relación entre LMA elevados en esclerófilos perennifolios y baja inversión de $\mathrm{N}$ en componentes fotosintéticos (Field y Mooney, 1986) que podria ser responsable del incremento de la limitación no estomática de la fotosintesis bajo estrés severo tal como se observó en los controles.

Se estudió la relación entre LMA y sus dos componentes: densidad foliar (D) y grosor (T) ya que pueden variar independientemente en respuesta a la disponibilidad hidrica, nutricional y luminosa (Witkowski y Lamont 1991). LMA no varió durante el estudio, pero si sus componentes en la vegetación de rebrote: $\mathrm{D}$ disminuyó y $\mathrm{T}$ aumentó de primavera a verano al aumentar la sequía (Figura $\mathrm{N}^{\circ} 4$ ). Mediavilla et al. (2001) mostraron el hecho de que hojas más gruesas tienden a mostrar una menor densidad de tejido. Las variaciones observadas de los componentes de las paredes celulares (hemicelulosa, celulosa y lignina) (Cuadro $\mathrm{N}^{\circ} 3$ ), siguen el mismo patrón que la densidad foliar: menores contenidos de celulosa y lignina en los rebrotes que en los controles en primavera y verano. 


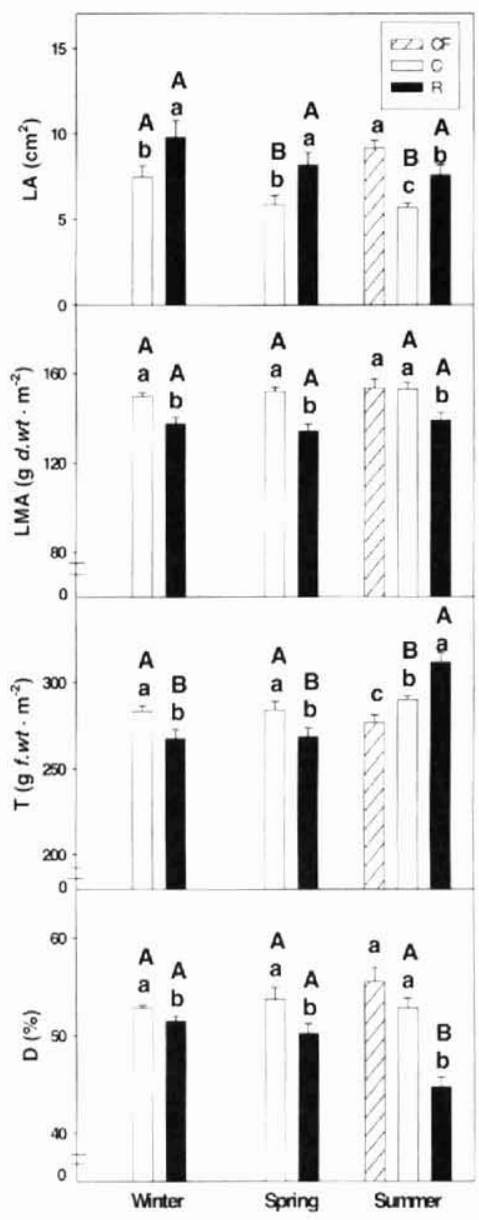

Cada valor representa la media \pm SE de 30 medidas

Las diferencias significativas ( $p \leq 0.05)$ se indican mediante letras distintas (tratamiento $a, b, c$, estación A, B)

\section{Figura $\mathrm{N}^{\circ} 4$}

\section{ÁREA MEDIA POR HOJA (LA), PESO ESPECIFICO FOLIAR (LMA), DENSIDAD FOLIAR} (D) Y GROSOR FOLIAR (T); (CF CONTROL BOSQUE; C CONTROL Y R, REBROTES).

Los cambios estructurales pueden favorecer una mayor $\mathrm{g}_{\text {mes }}$ en los rebrotes facilitando la difusión de $\mathrm{CO}_{2}$ en el mesófilo y contribuyendo al aumento fotosintético. En efecto, $\mathrm{g}_{\text {mes }}$ se correlacionó negativamente con $\mathrm{D}$ y positivamente con T (Figura $\mathrm{N}^{\circ} 5$; Niinemets 1999). Los valores de $\mathrm{g}_{\text {mes }}$ se redujeron con el estrés hídrico desde invierno a verano (Figura $\mathrm{N}^{\circ}$ 6) tal como se ha descrito en otras especies (Renou et al., 1990; Flexas et al., 2002), y se observaron variaciones a lo largo del dia. Tanto las variaciones estacionales como diurnas 
de $g_{\text {mes }}$ transcurrieron en paralelo a los cambios en A y Amax, hecho que podria indicar una regulación a la baja de la fotosíntesis en respuesta a niveles sostenidos y bajos de $\mathrm{CO}_{2}$ (Flexas et al., 2006). Los valores bajos de $g_{\text {mes }}$ respecto a los de $g_{s}$ sugieren una fuerte contribución de $\mathrm{g}_{\text {mes }}$ al declive general de la difusión de $\mathrm{CO}_{2}$ durante la sequia.

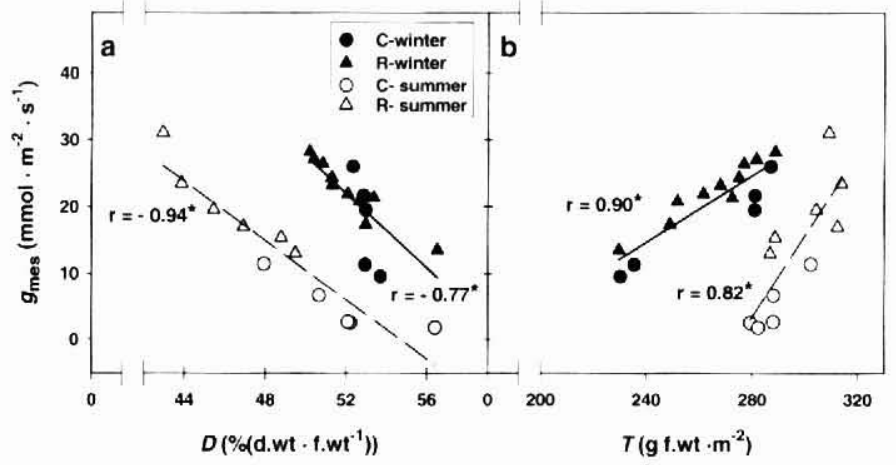

Los asteriscos indican correlaciones estadisticamente significativas $(p \leq 0.05)$

Figura $\mathrm{N}^{\circ} 5$

CONDUCTANCIA DEL MESÓFILO AL $\mathrm{CO}_{2}\left(\mathrm{G}_{\text {MES }}\right)$ VS. DENSIDAD FOLIAR (D) (A) Y GROSOR FOLIAR (T) (B) EN INVIERNO (NEGRO) Y VERANO (BLANCO).

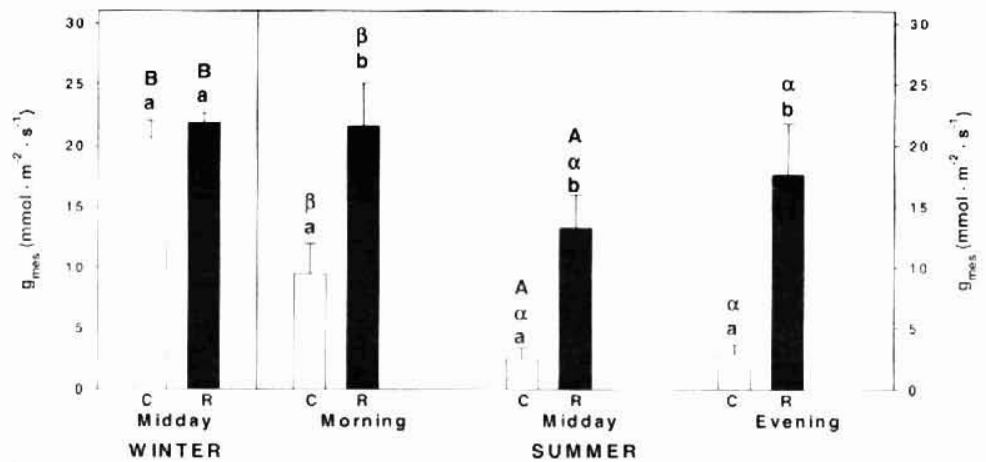

En verano se consideró también el momento del dia.

Cada valor representa la media \pm SE de 30 medidas.

Las diferencias significativas $(p \leq 0.05)$ se indican mediante letras distintas

(tratamiento ( $a, b, c)$, estación ( $A, B)$ y momento del dia (a. $\beta)$ ).

Figura $\mathrm{N}^{\circ} 6$.

CONDUCTANCIA DEL MESÓFILO AL $\mathrm{CO}_{2}\left(\mathrm{G}_{\mathrm{MES}}\right)$ (C, CONTROL Y R, REBROTES)

A pesar de la relación observada entre $g_{\text {mes }}$ y $\mathrm{D}$ y $\mathrm{T}$, se observó la presencia de dos correlaciones diferentes en invierno y verano, indicativa de la existencia de otro factor subyacente a las variaciones de $\mathrm{g}_{\text {mes }}$. Además, las variaciones observadas de $\mathrm{g}_{\text {mes }}$ a lo largo del dia no pueden ser explicadas por variaciones en la morfología foliar, sino que deberían atribuirse a 
cambios fisiológicos y/o bioquimicos. Tanto la anhidrasa carbónica (Gillon y Yakir, 2000), como las acuaporinas cloroplásticas (Terashima y Ono, 2002, Flexas et al., 2004 y Hanba et al., 2004) podrian jugar un papel regulador importante de la $g_{\text {mes }}$.

\section{CONCLUSIONES}

Los rebrotes de encina presentaron una mayor actividad fotosintética y mejor estado hidrico en época de sequía. Esta mejora funcional está asociada a cambios en la estructura y composición química foliar que conllevaron valores mayores de conductancia del mesófilo al $\mathrm{CO}_{2}$ y como resultado una mayor capacidad fotosintética que pueda contribuir al mejor comportamiento tras una perturbación en zonas propensas a la sequia.

\section{REFERENCIAS}

Chaves, M.M, Maroco, J.P., Pereira, J.S., 2003. Understanding plant responses to droughtfrom genes to the whole plant. Functional Plant Biology 30, 239-264

Demmig-Adams, B,, Adams, III W.W., 1996. Xanthophyll cycle and light stress in nature: uniform response to excess direct sunlight among plant species. Planta 198, 460-470

Dijkstra, P., 1989. Cause and effect of differences in specific leaf area. In: Lambers $\mathrm{H}$ et al. (eds) Causes and consequences of variation in growth rate and productivity of higher plants. SPB Academic Publishing bv, The Hague, The Netherlands, pp 125-140

Epron, D., Godard, D., Cornic, G., Genty, B., 1995. Limitation of net $\mathrm{CO}_{2}$ assimilation rate by internal resistances to $\mathrm{CO}_{2}$ transfer in the leaves of two tree species (Fagus sylvatica $\mathrm{L}$. and Castanea sativa Mill.). Plant Cell and Environment 18, 43-51

Farquhar, G.D., Sharkey, T.D., 1982. Stomatal conductance and photosynthesis. Annual Review Plant Physiology 33, 317-345.

Field, C., Mooney, H.A., 1986. The photosynthesis-nitrogen relationships in wild plants. In: Givinish TJ (ed) On the economy of plant form and function. Cambridge University Press, Cambridge, England, pp 25-56

Fleck, I., Grau, D., Sanjosé, M. and Vidal, D., 1996. Influence of fire and tree-fell on physiological parameters in Quercus ilex resprouts. Annals of Forest Science 53, 337-346

Fleck, I., Hogan, K.P., Llorens, L., Abadia, A. and Aranda, X., 1998. Photosynthesis and photoprotection in Quercus ilex resprouts after fire. Tree Physiology 18,607-614

Flexas, J., Bota, J., Escalona, J.M., Sampol, B. and Medrano, H., 2002. Effects of drought on photosynthesis in gravepines under field conditions: an evaluation of stomatal and mesophyll limitations. Functional Plant Biology 29, 461-471

Flexas, J., Bota, J. Loreto, F., Cornic, G. and Sharkey T.D., 2004. Diffusive and metabolic 
limitations to photosynthesis under drought and salinity in $C_{3}$ plants. Plant Biology 6, 269-279.

Flexas, J., Ribas-Carbó. M., Bota, J., Galmés, J., Henkle, M., Martinez-Cañellas, S., Medrano, H., 2006. Decreased Rubisco activity during water stress is not induced by decreased relative water content but related to conditions of low stomatal conductance and chloroplast $\mathrm{CO}_{2}$ concentration. New Phytol. Doi: 10.1111/j.1469-8137.2006.01794.x

Genty, B., Briantais, J.M. and Baker, N.R., 1989. The relationship between the quantum yield of photosynthetic electron transport and quenching of chlorophyll fluorescence. Biochemical Biophysical Acta 990, 87-92

Gillon, Jim and Yakir, Dan, 2000. Internal Conductance to $\mathrm{CO}_{2}$ Diffusion and $\mathrm{C}^{18} \mathrm{OO}$ Discrimination in C ${ }_{3}$ Leaves Plant Physiology 201-214.

Gratani, L. and Bombelli, A., 1999. Leaf anatomy, inclination, and gas exchange relationships in evergreen sclerophyllous and drought semideciduous shrub species. Photosynthetica 37. 573-585

Gulias, J., Flexas, J., Mus, M., Cifre, J., Lefi, E. and Medrano, H., 2003. Relations between maximum leaf photosynthesis, nitrogen content and specific leaf area in Balearic endemic and non-endemic Mediterranean species. Annals of Botany 92,215-222

Hanba, Y.T., Shibasaka, M., Hayashi, Y., Hayakawa, T., Kasamo, K., Terashima, I., Katsuhara, M., 2004. Overexpression of the barley aquaporin HvPIP2; 1 increases internal $\mathrm{CO}_{2}$ conductance and $\mathrm{CO}_{2}$ assimillation in the leaves of transgenic rice plants. Plant Cell Physiol. $45,21-529$.

Houghton, J.T., Ding, Y., Griggs, D.J., Noguer, M., van der Linden, P.J., Drai, X., Maskell, K. and Johnson, C.A. eds., 2001. Climate change: The Scientific Basis. Contribution of Working Group I in the Third Assessment Report of Intergovernmental Panel on Climate Change. Cambridge University Press, Cambridge, 251p

Kooten, O.V., Snel, J.F.H., 1990. The use of chlorophyll fluorescence nomenclature in plant stress physiology. Photosynthesis Research 25, 147-150.

Kruger, E.L. and Reich, P.B., 1997. Response of hardwood regeneration to fire in mesic forest openings. II Leaf gas exchange, nitrogen concentration and water status. Canadian Journal of Forest Research 27, 18321840

Loreto, F., Di Marco, G., Tricoli, D. and Sharkey, T.D., 1994. Measurements of mesophyll conductance, photosynthetic electron transport and alternative electron links of field grown wheat leaves. Photosynthesis Research 41, 397-403

Lawlor, D.W. and Cornic, G., 2002. Photosynthetic carbon assimilation and associated metabolism in relation to water deficits in higher plants. Plant Cell and Environment 25, 275294 
Mc Murtrie, R.E. and Wang, Y-P., 1993. Mathematical models of the photosynthetic responses of tree stand to rising $\mathrm{CO}_{2}$ concentrations and temperatures. Plant Cell Environment 16, 1-13

Mediavilla, S., Escudero, A. and Heilmeier , H., 2001. Internal leaf anatomy and photosynthetic resource-use efficiency: interspecific and intraspecific comparisons. Tree Physiology 21, 251 259

Méthy, M., Damesin, C. and Rambal, S., 1996. Drought and photosystem II activity in two Mediterranean oaks. Annals Forest Science 53, 255-262

Mouillot, F., Rambal, S. and Joffre, R., 2002. Simulating climate change impacts on fire frequency and vegetation dynamics in a Mediterranean-type ecosystem. Global Change Biology $8,423-437$

Niinemets, Ü., 1999. Research review. Components of leaf mass per area -thickness and density- alter leaf photosynthetic capacity in reverse directions in woody plants. New Phytologist $144,35-47$

Niinemets, Ü., 2001. Global-scale climatic controls of leaf mass per area, density, and thickness in trees and shrubs. Ecology 82, 453-469

Niinemets, U., Cescatti, A., Rodeghiero, M. and Tosens, T., 2005. Leaf internal diffusion conductance limits photosynthesis more strongly in older leaves of Mediterranean evergreen broad-leaved species. Plant Cell Environment 28: 1552-1566.

Oxborough, K. and Baker, N.R., 1997. Resolving chlorophyll a fluorescence images of photosynthetic efficiency into photochemical and non-photochemical components- calculation of $\mathrm{q}_{p}$ and $\mathrm{F}^{\prime} / \mathrm{F}_{m}^{\prime}$ without measuring $\mathrm{F}_{\mathrm{o}}^{\prime}$. Photosynthesis Research 54, 135-142

Peña-Rojas, K., Aranda. X. and Fleck, I., 2004. Stomatal limitation to $\mathrm{CO}_{2}$ assimilation and down-regulation of photosynthesis in Quercus ilex L. resprouts under slowly-imposed drought. Tree Physiology 24, 813-822

Peña-Rojas, K., Aranda, X., Joffre. R. and Fleck, I., 2005. Leaf morphology, photochemistry and water status changes in resprouting Quercus ilex during drought. Functional Plant Biology $32,117-130$.

Renou, J.L., Gerbaud, A., Just, D. and André, M., 1990. Differing substomatal and chloroplastic concentrations in water-stressed wheat. Planta 182, 415-419.

Saruwatari, M.W. and Davis, S.D., 1989. Tissue water relations of three chaparral shrub species after wildfire. Oecologia 80, 303-308

Tenhunen, J.D., Pearcy, R.W. and Lange, O.L., 1987. Diurnal variations in leaf conductance and gas exchange in natural environments. In: Zeiger E, Farquhar GD, Cowan IR (eds) Stomatal function. Stanford University Press, Stanford, California, USA, pp 323-352 
Terashima, I., Miyazawa. S.I. and Hanba ,Y.T., 2001. Why are sun leaves thicker than shade leaves?-consideration based on analyses of $\mathrm{CO}_{2}$ diffusion in the leaf. Journal of Plant Research $114,93-105$

Witkowski, E.T.F. and Lamont, B.B., 1991. Leaf specific mass confounds leaf density and thickness. Oecologia $88,486-493$ 